\title{
1 MRSA emerges through natural transformation
}

2 Mais Maree ${ }^{1, \#}$, Le Thuy Thi Nguyen 2,3,\#, Ryosuke L. Ohniwa ${ }^{4,5, \#, ~ S h e n g h e ~ H u a n g ~}{ }^{1}$, Masato

3 Higashide $^{6}$, Tarek Msadek ${ }^{4,7, \dagger}$, Kazuya Morikawa ${ }^{5, \dagger}$

4

$5 \quad{ }^{1}$ Graduate School of Comprehensive Human Sciences, University of Tsukuba, Japan

$6 \quad{ }^{2}$ Human Biology Program, School of Integrative and Global Majors, University of Tsukuba, Japan

$7 \quad{ }^{3}$ Biotechnology Centre of Ho Chi Minh City, District 12, HCM City, Vietnam

$8 \quad{ }^{4}$ Biology of Gram-Positive Pathogens, Department of Microbiology, Institut Pasteur, Paris, France

$9 \quad{ }^{5}$ Division of Biomedical Science, Faculty of Medicine, University of Tsukuba, Japan

$10 \quad{ }^{6}$ Kotobiken Medical Laboratories, Inc., Kamiyokoba, Tsukuba, Japan

$11{ }^{7}$ ERL3526, CNRS, Paris, France

12

13 \# these authors contributed equally to this work

$14{ }^{\dagger}$ these authors share last author ship

16 Key words

17 Staphylococcus aureus, MRSA, SCC (staphylococcal cassette chromosome), antimicrobial resistance,

$18 m e c A$, natural genetic competence, transformation, horizontal gene transfer, biofilm 


\section{Abstract}

Methicillin-resistant Staphylococcus aureus (MRSA) carries the resistance gene mecA in the staphylococcal cassette chromosome (SCC) that disseminates among staphylococci but the cell-to-cell transmission mechanism of SCC has not been clarified for half a century ${ }^{1}$. Here, we present evidence for efficient natural transformation in Staphylococcus aureus and its relevance in $\mathrm{SCCmec}$ transmission. We found that growth in biofilm conditions increased the transformation efficiency in a dependent manner on two component signal transduction systems, TCS13 (AgrCA) and TCS17 (BraSR). Strikingly, we demonstrate that natural transformation mediates the transfer of SCCmec from MRSA or methicillin-resistant coagulase negative staphylococci to methicillin-sensitive $S$. aureus. The site-specific insertion/excision system mediated by cassette chromosome recombinases was essential for SCCmec transformation while the stability of SCCmec varied depending on SCC types and recipients. We propose that natural transformation is the key process in the emergence of MRSA.

\section{Introduction}

Staphylococcus aureus is a Gram-positive bacterium belonging to Phylum Firmicutes that is resident in the nasal cavities of about 30 percent of the entire population. These $S$. aureus carriers are normally asymptomatic but opportunistic infections, ranging from minor skin abscesses to severe diseases (such as pneumonia, osteomyelitis, or toxic shock syndrome), are possible. Immunocompromised hosts are vulnerable but the spread of community-associated infections by highly virulent $S$. aureus has also been reported ${ }^{2}$.

Antibiotic resistance is the most notorious feature of this pathogen, especially methicillin-resistant $S$. aureus (MRSA). MRSA is the leading cause of nosocomial infections (health-care-associated MRSA; HA-MRSA) and is also associated with healthy individuals 
(community-associated MRSA; CA-MRSA) and livestock (livestock associated MRSA; LAMRSA), posing a global health burden ${ }^{3,4,5,6}$. The percentage of MRSA among $S$. aureus isolates is diverse among countries (Vietnam 73\%, United States 45\%, Japan 41\%, North Europe 1\%), raising concerns in clinics, care homes and other places with high densities of immunocompromised individuals ${ }^{7}$.

virulence factors and antibiotic resistance genes, many of them located on mobile genetic elements (MGEs) such as plasmids, prophages, transposons, pathogenicity islands, insertion sequences, and the staphylococcal cassette chromosome (SCC) ${ }^{8,9}$. In MRSA and methicillinresistant coagulase negative staphylococci (MR-CoNS), the methicillin-resistant determinant $m e c A$ is always located within the $\mathrm{SCC}$ (SCCmec). SCCmec is itself a 20-60 kb genetic element integrated by Ccr recombinases at a specific site $(a t t B)$ in $\operatorname{orf} X$ near the replication origin of the chromosome ${ }^{10}$. Epidemiological studies show that these SCCmec elements are transmitted among staphylococci and at least 20 independent acquisitions of SCCmec were reported to have occurred in $S$. aureus ${ }^{11}$, but the exact mechanism of cell-to-cell transmission has been debated for over 50 years ${ }^{1}$ (see Discussion).

The presence of diverse MGEs conveying virulence and resistance factors to the $S$. aureus genome indicates a prominent evolutionary ability mediated by horizontal gene transfer (HGT). Bacteriophage-mediated transduction and conjugative machinery-dependent conjugation are historically well-characterized HGT mechanisms in staphylococci, with the former considered to be the primary method $^{1}$. In 2012, a subpopulation of $S$. aureus was shown to develop natural genetic competence for DNA transformation by expressing competence machinery (DNA incorporation machinery) genes in the com $G$ and comE operons, which are under the direct transcriptional control of the 'cryptic' sigma factor

$77 \mathrm{SigH}^{12,13}$. Bacteria modified to overexpress $\mathrm{SigH}$ incorporated plasmid DNA as well as 
78 SCCmec II elements ${ }^{13}$. However, the transformation efficiencies of the unmodified model strains (N315 or N315ex) and other clinical isolates in that report were under the detection limit $\left(<10^{-11}\right)^{13}$. Competence transcription factor ComK was also shown to synergistically upregulate many competence genes ${ }^{14}$. However, efforts to transform the tested strains by overexpressing SigH and ComK was unsuccessful ${ }^{14}$. These observations have led to the current belief that natural transformation plays no role in staphylococcal evolution, including the multiple, independent emergence of MRSA strains with diverse genetic backgrounds ${ }^{15}$.

In the present study, we identify specific two-component systems (TCSs) ${ }^{16,17}$ involved in the regulation of the competence operon promoter $\left(\mathrm{P}_{c o m G}\right)$. As TCSs are major mediators of sensing and environmental response, we conducted a survey to clarify the conditions that trigger efficient transformation in $S$. aureus through this mechanism. Strikingly, we present the first experimental evidence of efficient inter- and intraspecies transfer of SCCmec among staphylococci. We propose natural transformation as a major evolutionary strategy for this ever-adaptive pathogen.

\section{Results}

\section{TCSs are involved in the expression of $\operatorname{com} G$ promoter in subpopulations}

To delineate conditions conducive to natural transformation, we generated a series of 15 TCS deletion mutants, removing each TCS $(\Delta 3 \sim \Delta 17)$ except the essential TCS1 $(\mathrm{WalKR})^{18}$, in the S. aureus strain N315ex w/o $\phi^{13} \quad$ (termed Nef; Supplementary Table 1) (Fig. 1a) . Nef is an N315 derivative strain that can develop natural genetic competence but does not possess any conjugative elements or a lysogenic phage that transfers DNA by transduction or pseudo-competence. Nef also lacks the SCCmec and its embedded TCS2 (SA0066-SA0067). 

using CS2 medium, the GFP intensity of the parental strain (Nef-GFP) initially increases from $8 \mathrm{~h}$ and peaks around $15 \mathrm{~h}$ (Supplementary Fig. 1a). In contrast, no GFP could be detected in other media such as TSB (Supplementary Fig. 1b), in line with our previous observations that the activation of the $\operatorname{com} G$ promoter is dependent upon culture conditions ${ }^{13}$. significant effect. We also used fluorescence microscopy to observe cell populations expressing the GFP reporter (Fig. 1c) and, in the parental strain, 11.3\% of the cells expressed GFP after culturing for $12-14 \mathrm{~h}$ in CS2 medium but, in $\Delta 13$ and $\Delta 17$, only $2.9 \%$ and $0.1 \%$ of the cells expressed GFP (Fig. 1c, middle and right panels). In $\Delta 12$, on the other hand, $49.3 \%$ of the cells expressed GFP, an approximately 4-fold higher expression than the parental strain of GFP-positive cells towards the parental strain levels. Taken together, our results suggest the involvement of TCS12, TCS13, and TCS17 in the regulation of natural genetic competence in Nef (Fig. 1d).

\section{Cell wall-targeting antibiotics and biofilm growth conditions modulate com $G$ expression}


involved in resistance to bacitracin and $\operatorname{nisin}^{20}$. Indeed, we confirmed that $\Delta 12$ is susceptible

130 to vancomycin and $\Delta 17$ is susceptible to bacitracin and nisin in CS2 and TSB media

131 (Supplementary Table 3).

In addition to their essential roles in resistance to cell wall-targeting antibiotics,

TCS17 has pleiotropic roles in cell physiology, including biofilm formation ${ }^{23}$, and TCS13

(AgrCA) is a part of the accessory gene regulator (Agr) quorum sensing system that regulates the expression of multiple virulence genes ${ }^{24}$ by the diffusion sensing mechanism ${ }^{25,26}$ and is also involved in biofilm regulation. We noted that $\Delta 13$ and $\Delta 17$ were impaired in rigid biofilm formation compared to Nef when cultured in CS2 medium (Supplementary Fig. 3) but this phenotype is not due to growth defects as the colony forming units of $\Delta 13$ and $\Delta 17$ were not significantly different compared to Nef after $24 \mathrm{~h}$ under these static conditions (Supplementary Fig. 3b, right). Based on these results, we tested the effects of cell walltargeting antibiotics and biofilm-forming, static growth conditions on $\mathrm{P}_{c o m G}-g f p$ expression. bacitracin reduced $\mathrm{P}_{c o m G}-g f p$ reporter expression in a concentration-dependent manner 144 (Supplementary Figs. 4a,b). On the other hand, 8 or $16 \mu \mathrm{g} \mathrm{mL}{ }^{-1}$ nisin slightly, but reproducibly, increased reporter expression intensity in Nef-GFP (Supplementary Fig. 4c).

To test bacteria under biofilm growth conditions, we cultured Nef and its derivative strains statically in CS2 medium to let cells sediment at the bottoms of a 6-well polystyrene plate where they stably attach by forming a biofilm (Supplementary Fig. 3b). In the Nef-GFP reporter strain, the percentage of GFP-expressing cells increased towards days 2-3 (Fig. 2a),

150 an effect that was impaired in $\Delta 13$ and $\Delta 17$. The percentage of GFP-expressing cells in

151 biofilm was less than in planktonic culture, but our data indicate that the Agr quorum sensing 152 TCS13 plays a role in $\operatorname{com} G$ reporter expression under biofilm-forming conditions. 153 Furthermore, we examined if other TCSs are involved in $\mathrm{P}_{c o m G}-g f p$ expression under these 
same conditions and found that only $\Delta 13$ and $\Delta 17$ had significantly reduced GFP expression compared with Nef after 3 days (Supplementary Fig. 5) whereas $\Delta 7, \Delta 9$, and $\Delta 12$ had significantly increased GFP expression. Complementation in $\Delta 13$ and $\Delta 17$ restored $\mathrm{P}_{c o m G}-g f p$ expression to levels equivalent to Nef values in the biofilm (Fig. 2b).

In conclusion, these data indicate that $\mathrm{P}_{c o m G^{-}} g f p$ expression is affected by cell walltargeting antibiotics as well as environmental cues or cellular status in biofilm where TCS13 and TCS17 play important regulatory roles.

\section{Biofilm growth conditions induce efficient natural transformation}

Nisin and biofilm conditions were tested with tetracycline-resistant donor cells $\left(\mathrm{N} 315 \Delta \mathrm{cls} 2\right.$, tet ${ }^{\mathrm{R}}$, or Nef $\Delta \mathrm{cls} 2$, tet $\left.{ }^{\mathrm{R}}\right)$ for their effects on transformation efficiencies. Nisin $\left(8 \mu \mathrm{g} \mathrm{mL} \mathrm{m}^{-1}\right)$ had no detectable effect on transformation in Nef planktonic growth (Supplementary Fig. 6) and transformation was unaffected in the SigH-overexpressing strain (Nefh). Under biofilm-forming conditions, however, the transformation frequency in Nef increased, reaching $10^{-6 \sim 7}$ at day 3 (Fig. 2c), which was remarkably higher than in the planktonic growth condition (undetected, $<10^{-11}, \mathrm{n}=5$ ) (Fig. 2c). In Nefh, the transformation frequency was similar to Nef under biofilm-forming conditions in that it was higher than in the planktonic growth condition $\left(\sim 10^{-11}, \mathrm{n}=5\right)$ (Fig. $\left.2 \mathrm{c}\right)$.

Figure $2 \mathrm{~d}$ shows the time course for the transformation frequencies of Nef, Nefh, $\Delta 13, \Delta 17, \mathrm{Nef} \Delta \mathrm{comG}$, and Nef $\Delta$ comE. Unexpectedly, we found that one of the negative control strains, the $\triangle \operatorname{com} G$ operon, was also transformable in biofilm while the $\Delta c o m E$ operon was not (Fig. 2d). This observation is consistent with reports that the comE operon encodes an essential DNA incorporation channel while the $\operatorname{com} G$ operon encodes the pseudopilin that facilitates DNA access to this channel ${ }^{27}$. Transformation frequencies of $\Delta 13$ 
and $\Delta 17$ were significantly reduced at day 3 compared to Nef (Fig. 2e) but complementation restored transformation frequencies in biofilm to levels equivalent to Nef (Fig. 2e). for detecting transformation. We therefore evaluated if static biofilm conditions could induce natural transformation with other growth media such as TSB, BHI, RPMI, or M9 in Nef and Nefh in all growth media but the efficiency was 100 to 1000 -fold lower than in CS2 medium, indicating that CS2 medium is dispensable but preferable for efficient natural transformation in Nef biofilm.

\section{Clinical isolates are capable of natural transformation in biofilm}

As natural transformation in $S$. aureus has only been detected in N315 derivative strains that were genetically engineered to express $\mathrm{SigH}^{13}$, we tested the transformability of 5 unmodified clinical isolates (tetracycline susceptible) by employing the biofilm conditions described above. We found that one strain (MRSA, r59) was transformable by the tetracycline resistance marker (Supplementary Fig. 8) whereas other strains became transformation competent after introducing a SigH-expressing plasmid (pRIT-sigH), suggesting that $\operatorname{sig} H$ expression was still a limiting step in the transformation of MSSA s142 and MRSA r3. Two strains (MSSA s1567 and MRSA r408) were not transformable irrespective of the SigH-expressing plasmid. Taken together, these data reveal that the biofilm condition facilitates transformation in some, but not all, clinical isolates.

\section{SCCmec elements can be transferred in biofilm}

Exploring the SCC transfer mechanism is challenging as small SCC elements can be 
203 Siphoviridae with genome sizes of less than c.a. $45 \mathrm{~kb}^{29}$ and cannot physically accommodate 204 an entire large $\mathrm{SCC}^{30}$. Conjugation can also transfer a shortened SCCmec II but this requires insertion of SCC into the conjugative plasmid ${ }^{31}$ and, to the best of our knowledge, such a plasmid-carrying SCC has not been reported in staphylococcal isolates. In this study, we propose that natural transformation in biofilms is the major mechanism for SCC transfer based on the following experimental evidence.

We tested the natural transformation of $m e c A$ in biofilms by using clinical isolates of MSSA as recipients and heat-killed MRSA or methicillin-resistant coagulase-negative staphylococci strains (MR-CoNS) as donors. The mecA transformants were selected by cefmetazole (from the cephem subgroup of the $\beta$-lactam antibiotics). We first tested 20 MSSA clinical isolates using MR-CoNS8 as donor (for 1s- 20s) and other 20 MSSA using MR-CoNS3 as donor (21s- 40s), and found that 6 strains were able to form colonies on cefmetazole plates. Among these 6 strains, $4(1 \mathrm{~s}, 9 \mathrm{~s}, 11 \mathrm{~s}, 35 \mathrm{~s})$ were selected for further analysis (Supplementary Table 4). These 4 MSSA strains, together with Nef and Nef $\Delta$ comE as positive and negative controls, were tested for their transformability with distinct staphylococcal species and SCC types. Either S. aureus (including 35 transformants; 35s

219 [CoNS17]) or MR-CoNS, along with any tested SCCmec (I, II, III, IVa), could serve as the donor with the detected efficiencies ranging from c.a. $10^{-8}$ to $10^{-7}$, generating up to $\sim 160$ colonies from a single-well biofilm containing $10^{9}$ c.f.u recipient cells. To confirm this observation as natural transformation, we deleted the comE operon of $9 \mathrm{~s}$ and found that this mutant was non-transformable using N315 as the donor (Supplementary Table 4). Some of these MSSA recipients $(1 \mathrm{~s}, 9 \mathrm{~s}, 11 \mathrm{~s}$, but not $35 \mathrm{~s})$ were transformed by the chromosomal tetracycline resistance gene while no transformation was detected using the pT181 plasmid in any transformable strain (Supplementary Table 4, Nef-pT181). The 
question as to transfer of other plasmids by natural transformation under biofilm conditions remains unanswered.

Figure 3a shows the result of mecA colony PCR for multiple transformants. All transformants derived from $35 \mathrm{~s}$ showed the mecA signal and the minimum inhibitory concentrations (MICs) of cephems (cefmetazole and cefoxitin) were increased in these transformants, demonstrating their conversion to MRSA (Supplementary Table 5). In contrast, some transformants showed lower intensities in mecA signal compared with $35 \mathrm{~s}$ transformants (Fig. 3a). Moreover, the MIC values in these transformants were relatively lower than $35 \mathrm{~s}$ transformants, suggesting that $35 \mathrm{~s}$, but not others, could stably accommodate the mec $A$ gene. The stability test of cefoxitin resistance showed that the $35 \mathrm{~s}$ transformant $(35 \mathrm{~s}$ [CoNS15]) sustained the resistance in the absence of $\beta$-lactam but $1 \mathrm{~s}, 9 \mathrm{~s}$, and $11 \mathrm{~s}$ derivatives tested swiftly lost their resistance (Fig. 3b). Disk diffusion test (Fig. 3c) confirmed the reduced susceptibility of the stable transformant $35 \mathrm{~s}[\mathrm{CoNS} 15]$ to cefoxitin (CFX) and oxacillin (MPI). On the other hand, the unstable 9s[CoNS15] was categorized into the MSSA criteria, though the inhibitory zone of CFX slightly decreased and colony appeared on the edge of the inhibitory zone of MPI. Notably, we detected stable 9s transformants when N315 was used as donor. This suggests that SCCmec type and recipient strain are drivers of

244 SCCmec stability in transformants. PCR analysis showed that the full-length transferred 245 SCCmec IVa was present in the stable 35s transformants (Fig. 3d). Full-size SCCmec II was 246 detected in transformants of Nef and 9s but was shortened in 35s transformants, possibly due

247 to the elimination of mobile elements Kdp, Tn554, and IS431-pUB110 (Fig. 3e). Stability and SCC intactness in transformants are summarized in Fig. 3f. Collectively, these observations demonstrate that SCCmec elements can be transferred to MSSA strains by natural transformation in biofilm. 
SCCmec transformation depends on $\operatorname{ccr} A B$ recombinase genes and an intact att $B$ site determine whether SCCmec transformation is mediated by CcrAB (cassette chromosome recombinases) or general homologous recombination, we deleted the $\operatorname{ccr} A B$ gene from the

SCCmec II element (N315 $\Delta \mathrm{ccr} A B)$ and evaluated its ability to serve as SCCmec donor for 9s and Nef strains. SCCmec transformants could not be obtained using N315 $\Delta$ ccrAB as a donor (Supplementary Table 4), suggesting that the site-specific excision and integration of SCCmec mediated by $c c r A B$ is essential for transformant generation. We also generated SCCmec transformants when N315 was used as donor (Supplementary Table 4). This novel evidence points to the $c c r A B$-attB-dependent SCC transfer system as critical for SCCmec transformation.

\section{Bacitracin inhibits natural transformation}

Our finding that cell wall-targeting antibiotics affect $\mathrm{P}_{c o m G}$ activity (Supplementary

Fig. 4) suggests that they may also affect natural transformation. To test this point, we treated cells growing in biofilm with either bacitracin, vancomycin, or nisin for three days. Lowconcentration bacitracin treatment $\left(0.5 \mu \mathrm{g} \mathrm{mL}^{-1}\right)$ reduced the transformation efficiency while $5 \mu \mathrm{g} \mathrm{mL} \mathrm{m}^{-1}$ completely prevented the detection of transformants in Nef (Fig. 4a). Vancomycin treatment, on the other hand, had no significant effect on natural transformation in this strain transformation efficiency (Fig. 4c). 


\section{Discussion}

This study demonstrated HGT of SCCmec by natural transformation and provides mechanistic information on the pathway of MRSA emergence. SCC, an MGE shared among staphylococcal species and Macrococcus caseolyticus ${ }^{32}$, is responsible for dissemination of virulence factors and resistance genes such as capsule synthesis genes (SCCcap) $)^{33,34}$, the fusidic acid resistance gene (SCCfus) ${ }^{35}$, and the methicillin resistance gene (SCCmec) (see comprehensive review ${ }^{36}$ ). Since its discovery, SCCmec has been a research focus of extensive efforts to clarify the global emergence and dissemination of MRSA. In 1961, the first MRSA, which carried the type I SCCmec, was isolated in the United Kingdom. Types II and III, identified in the early 1980s in Japan and New Zealand, were clinically isolated and are reported as the largest types among $\mathrm{SCCs}^{37,38}$. SCCmec IV and V were described in United States and Australia but are relatively small and found primarily in communityacquired MRSAs ${ }^{39,40}$. While Types I to V are dominant and widely distributed, diverse new variants have been reported (Types VI - XIII). The origins of SCCmec are unclear but ancestral forms have been identified in coagulase-negative staphylococci such as $S$. sciuri, $S$. fleuretti, S. xylosus, S. hominis, and M. caseolyticus ${ }^{32,41,42}$. Cor recombinases were found to mediate the excision and insertion of SCC at the attB locus (attL/attR after SCCmec integration $^{10}$, with CcrA and CcrB for SCC type I type V and CcrC for type V. These $\operatorname{ccr} A B$ genes are expressed in minor subpopulations and the excised circular SCC is thought to serve as a donor for horizontal transmission ${ }^{43}$. Both proteins are required for the proper excision of SCCmec from the chromosome and its integration into the attB site after transduction as a part of the artificial plasmid ${ }^{44}$.

Despite such well-established epidemiological evidence of interspecies SCCmec movement and $c c r$-dependent excision/insertion, the major intercellular transmission mechanism has remained enigmatic for half a century. Transfer of the methicillin resistance 
gene was first demonstrated by transduction ${ }^{45}$ and by pseudo-competence ${ }^{46}$ which, at that time was described as 'transformation' but, after discovery of the phage component, is now a preferable transfer route for some types of SCCmec (albeit with a $<45 \mathrm{~kb}$ capacity limit of the bacteriophage capsid) and SCCmec fragments are detectable in bacteriophage capsids ${ }^{47,48}$, making the transfer of short SCCmec (types IVa and I) observable by transduction among compatible strains ${ }^{28}$. However, major deletions are occasionally associated with transduction $^{28}$ and successful integration into the recipient chromosome requires homologous flanking sequences, suggesting that this transduction relies on homologous recombination rather than the ccr-mediated system. Conjugation has also been suggested as a possible mechanism for SCCmec transfer ${ }^{31}$. However, successful transfer requires donor manipulation by overexpressing the $c \mathrm{cr}$ recombinase to capture a shortened $\mathrm{SCCmec}$ into a conjugative

314 plasmid ${ }^{31}$ while spontaneous and large element transfers have not been demonstrated. excision/integration. To the best of our knowledge, this study is the first to show that natural intracellular SCC HGT requires the $c c r-a t t B$ system and, based on this evidence, we propose transformation efficiency, up to $10^{-7}$ (Supplementary Table 4), strongly supports the historical, independent transfers of distinct SCC types to $S$. aureus. Although transduction remains as a candidate HGT mechanism for short SCCmec, the extent of $c c r$ involvement in this process remains elusive. cost and the process of obtaining $\beta$-lactam resistance is complex, involving multiple mutations and metabolic adaptations ${ }^{49,50,51}$. It was previously suggested that different $S$. aureus genetic backgrounds offer different capacities to accommodate $m e c A^{11,52}$ and we 
observed a similar effect as two of our tested MSSA strains (9s and 35s) could stably accommodate the transferred mecA whereas 1s and 11s could not (Fig. 3). The methodology of SCCmec transfer established in this study would be invaluable to detail the genetic factors that define $m e c A$ stability.

The present study clarified that biofilm conditions are preferable for natural transformation in S. aureus. All tested factors that positively affect biofilm formation also increased transformation efficiency, such as CS2 medium, static growth (Fig. 2), TCS13, and TCS17 (Fig. 2), but how the biofilm structure increases transformation efficiency remains unknown. It is likely that transformation following competence gene expression is facilitated in biofilm as the $\mathrm{P}_{\text {comG }}$ reporter expression was reduced but transformation frequency was increased in biofilm compared to the planktonic state (Figs. 1,2). In addition, the transformation efficiency of SigH-overexpressing cells was higher in biofilm than in planktonic growth (Fig. 2c). In order to detect transformation in biofilm, however, it was crucial to use heat-killed donor cells rather than purified chromosomal DNA. This might be consistent with the fact that nuclease production is a common characteristic across all strains of $S$. aureus and also occurs in biofilms ${ }^{53}$. Alternatively, it is possible that experimentally added, purified DNA cannot serve as a transformation donor since extracellular DNA is known to be tightly sequestered in biofilm ${ }^{54,55}$. It is interesting to note that, in Streptococcus pneumoniae, non-competent cells undergo lysis by bacteriocins and fratricins released by neighboring competent cells ${ }^{56}$ but the presence of such a dedicated mechanism for DNA supply is not known in the Staphylococcus genus.

Staphylococcal infections are intimately associated with biofilm formation ${ }^{57}$ as it provides protection against antimicrobial treatment and host clearance mechanisms ${ }^{58}$ while contributing to the prolonged infection and colonization that facilitates the dissemination of drug-resistant strains $^{59}$. Our finding that $S$. aureus can develop natural transformation in 
biofilm conditions emphasizes the additional role of biofilms in promoting HGT as well as

353

354 transduction and conjugation ${ }^{1,60}$. Additionally, cells release phage at higher frequency than in planktonic conditions ${ }^{61}$ and subsequent cell lysis in biofilm would create an ample supply of genetic material for non-lysed cells. Interestingly, expressing SigH was shown to stabilize phage lysogeny ${ }^{62}$, implicating a co-evolution of distinct HGT mechanisms in staphylococcal biofilms. Mixed biofilms of $S$. aureus and other staphylococci formed during commensal state or co-infections are thus general hot spots for HGT.

Crucially, natural transformation can transfer longer DNA fragments, such as SCCmec II (Fig. 3e), that are too large to be packed into the typical staphylococcal bacteriophages ${ }^{13}$. Natural transformation cannot be abolished by inactivating the donor, unlike other HGT mechanisms such as phage transduction, conjugation, and the staphylococcal pathogenicity island-helper phage system. In order to counter staphylococcal evolution by SCC systems, specific control methods against transformation are therefore necessary and the recipient cell's signal transduction systems (e.g., TCS17) might serve as a promising target. The finding of the inhibitory effect of bacitracin (Fig. 4a) may also serve as an attractive future direction for experimental studies.

\section{Methods}

\section{Bacterial strains and culture conditions}

Bacterial strains and plasmids used in this study are listed in Supplementary Table 1. Clinical staphylococcal samples (40 MSSA isolates and 7 MR-CoNS isolates) were collected from the Kanto area of Japan. Unless otherwise indicated, staphylococci were grown in Trypticase Soy Broth (TSB). E. coli strains were grown in LB. Cultures were incubated at 
377

378

379

380

381

382

383

384

385

386

387

388

389

390

391

392

393

394

395

396

397

398

399

400

$37^{\circ} \mathrm{C}$ either with shaking (180 r.p.m) or statically. Where required for selection, culture medium was supplemented with chloramphenicol $(12.5 \mu \mathrm{g} \mathrm{mL}-1)$, kanamycin $\left(100 \mu \mathrm{g} \mathrm{mL}^{-1}\right)$, tetracycline $\left(5 \mu \mathrm{g} \mathrm{mL}^{-1}\right)$, cefoxitin $\left(4 \mu \mathrm{g} \mathrm{mL}^{-1}\right)$, cefmetazole $\left(4 \mu \mathrm{g} \mathrm{mL}^{-1}\right)$, or ampicillin $(100$ $\left.\mu \mathrm{g} \mathrm{mL} L^{-1}\right)$

\section{Construction of deletion and substitution mutants}

Each mutant was constructed from Nef or 9s by double-crossover homologous recombination using the pMADtet vector ${ }^{13}$ (Supplementary Table 1). Briefly, two fragments flanking the upstream (primers A, and B, Supplementary Table 2) and downstream (primers C, and D, Supplementary Table 2) regions of the locus targeted for deletion (or substitution) were amplified by PCR. The PCR products (AB and CD fragments) were used as template to generate the construct $\mathrm{AD}$ by overlapping PCR, using the primers $\mathrm{A}$ and $\mathrm{D}$ depending on locus (Supplementary Table 2). Product AD was cloned into the BamH I - Sal I site of pMADtet to generate the vectors for TCS deletions (pMADtet- $\Delta 3$ to $\Delta 17$ ), pMADtet- $\Delta$ ccrAB, and pMADtet-attB* (Supplementary Table 1). In terms of attB substitution, primers B and C were designed not to change the coding amino acid sequence of OrfX. The plasmids, purified from E.coli DH5 $\alpha$, were introduced into Nef, after passaging through RN4220. Mutants (tetracycline sensitive, $\beta$-galactosidase negative) were selected as described previously ${ }^{13,63}$ and the absence of the target gene was confirmed by PCR using the primers E and F (Supplementary Table 2). The attB substitution mutant was confirmed by restriction digestion (HindIII: included in the designed primers attB-B and attB-C) of the PCR product generated by primers E and F (Supplementary Table 2). Nef $\Delta$ cls 2 strain was created by transduction using the donor N315 $\Delta$ cls2 (carries a tetracycline resistance gene at the cls 2 locus ${ }^{64}$ ) (Supplementary Table 1).

401 


\section{Complementation of $\Delta \mathrm{TCSs}$}

For in trans complementation, each TCS gene (including its own promoter) was

404

405

406

407

408

409

410

411

412

413

414

415

416

417

418

419

420

421

422

423

424

425

426 amplified by PCR using chromosomal DNA from Nef as genomic template. The PCR product was cloned into the EcoR I - BamH I site of pHY300PLK (Takara) to generate the complementation plasmids pHY-12, pHY-13, and pHY-17 (Supplementary Table 1). These plasmids were introduced into the corresponding mutants after passaging through RN4220.

For chromosomal complementation of $\Delta 13$ and $\Delta 17$, each TCS its flanking region were amplified by PCR using primers G and H (Supplementary Table 2). Product was cloned into BamH I - Sal I site of pMADtet to generate the vectors pMADtet-13 and pMADtet-17 (Supplementary Table 1). The plasmids were purified from E.coli DH5 $\alpha$ and introduced into the corresponding $\triangle \mathrm{TCS}$ after passaging through RN4220. Complemented mutants (tetracycline sensitive, $\beta$-galactosidase negative) were selected as described previously ${ }^{13,63}$ and the presence of the restored gene was confirmed by PCR using the primers E and F (Supplementary Table 2).

\section{Antimicrobial susceptibility testing}

MIC assays were conducted in a 96-well microtiter plate (round bottom). Overnight bacterial cultures were diluted 1:2000 in appropriate medium and 100 $\mu \mathrm{L}$ aliquots were used to inoculate wells containing TSB or CS2 media supplemented with twofold serial dilutions of antibiotics (vancomycin, bacitracin, nisin, cefmetazole, or cefoxitin). The plates were statically incubated for $20 \mathrm{~h}$ at $37^{\circ} \mathrm{C}$. The MIC was determined by the lowest concentration of antibiotic at which growth was inhibited.

Disk diffusion testing was conducted according to CLSI standard using direct colony suspension method. Briefly, glycerol stocks of 9s, 35s, CoNS15, and 35s[CoNS15] were streaked on drug-free TSA. Unstable transformant $(9 \mathrm{~s}[\mathrm{CoNS} 15])$ was streaked on TSA 
427 supplemented with $4 \mu \mathrm{g} \mathrm{mL} \mathrm{mL}^{-1}$ cefoxitin. Emerged colonies were suspended in $0.85 \% \mathrm{NaCl}$ and turbidity was adjusted to $0.5 \mathrm{McF}$ arland standard. The inocula were swabbed on Mueller-

429 Hinton agar and the antibiotic disks of oxacillin $(1 \mu \mathrm{g})$, and cefoxitin $(30 \mu \mathrm{g})$ (KB disks, 430 Eiken Chemical) were used for susceptibility testing. Zone of inhibition was determined following $18 \mathrm{~h}$ of incubation at $35^{\circ} \mathrm{C}$.

\section{Measurement of $\operatorname{com} G$ promoter activity by a GFP reporter assay}

The reporter plasmid of $\mathrm{P}_{c o m G}$-gfp (pMK3-com-gfp) ${ }^{13}$ was introduced into each strain

by electroporation after passaging through RN4220. Reporter strains were grown overnight

vancomycin, bacitracin, or nisin as appropriate. Next, $200 \mu \mathrm{l}$ of these diluted cultures were

438 placed in a transparent, 96-well flat-bottomed microplate (Thermo Scientific, MA, USA) over 36 hours with 30 min intervals. The fluorescence intensity was normalized by the $\mathrm{OD}_{600}$ 442 value.

To count the numbers of GFP-expressing cells in planktonic culture, $50 \mu$ of overnight culture for each reporter strain was inoculated into $10 \mathrm{~mL}$ of CS2 medium in a glass vial. These cells were grown at $37^{\circ} \mathrm{C}$ with shaking for the appropriate time period

446 before $5 \mu \mathrm{L}$ of the culture was placed on slide, sealed with a cover glass and observed by the 447 fluorescence microscope (BZ-X710, Keyence). To count GFP-expressing cells grown in the static biofilm condition, bacteria in the biofilm were collected by extensive pipetting, washed and suspended in PBS, and stained by propidium iodide (40 $\mu \mathrm{M}$ final concentration) (WAKO) to distinguish dead cells (red fluorescence) from living cells. Stained bacteria were observed 
by the fluorescence microscope. The percentage of GFP-expressing cells was calculated by dividing the number of GFP-expressing cells by the total number of living cells.

\section{Natural transformation assay}

Donors used in natural transformation assays either include a purified plasmid ( $\mathrm{pHY}$ 300PLK) (Supplementary Fig. 6) or heat-killed cells (Figs. 2c,d,e, 3, 4, Supplementary Figs. suspended in $5 \mathrm{ml}$ PBS before heating in boiling water for $10 \mathrm{~min}$. The absence of viable cells was confirmed by plating on TSB agar. were carried out as previously described ${ }^{13}$ with some modifications. Briefly, $500 \mu 1$ of recipient cells from overnight cultures were washed and inoculated in $10 \mathrm{ml}$ CS2, supplemented with $8 \mu \mathrm{g} \mathrm{mL} \mathrm{m}^{-1}$ nisin when required, and were grown for $8 \mathrm{~h}$ at $37^{\circ} \mathrm{C}$ with shaking. Cells were then harvested and suspended in fresh $10 \mathrm{ml} \mathrm{CS} 2$ containing $10 \mu \mathrm{g}$ of purified pHY-300PLK plasmid (Supplementary Fig. 6) or $5 \times 10^{10}$ heat-killed N315 $\Delta$ cls2 cells transformants.

To detect natural transformation in biofilms (Fig. 2, 3, 4, Supplementary Figs. 7,8,

471 Supplementary Table 4), overnight cultures of recipient cells were diluted 1:200 in TSB and 472 grown with shaking for $3 \mathrm{~h}$ at $37^{\circ} \mathrm{C}$ before harvesting $750 \mu \mathrm{l}$ of the culture, washing it and 473 suspending it in appropriate growth medium (CS2 medium, TSB, BHI, RPMI 1640, or M9 474 supplemented with amino acids as in CS2 medium $\left.{ }^{13}\right)$. The $750 \mu 1$ of cell suspension ( $10^{8}$ c.f.u $475 \mathrm{~mL}^{-1}$ ) was distributed in a 6-well flat bottom polystyrene plate (Costar ${ }^{\circledR}$, Corning) and $250 \mu \mathrm{l}$ 
476 of heat-killed donor cells $\left(10^{9}\right.$ c.f.u $\left.\mathrm{mL}^{-1}\right)$ were added. The total volume of growth medium

477 was adjusted to $1.5 \mathrm{~mL}$ per well. The 6 -well plate was statically incubated at $37^{\circ} \mathrm{C}$ and

478

479 medium was refreshed every $24 \mathrm{~h}$. After incubation for an appropriate time period, the biofilm was harvested by extensive pipetting and was poured into BHI agar supplemented with $5 \mu \mathrm{g} \mathrm{mL} \mathrm{m}^{-1}$ tetracycline or $4 \mu \mathrm{g} \mathrm{mL}^{-1}$ cefmetazole, depending on the donor used. Unless otherwise indicated, all transformation frequencies from the assays were determined after 3 days of growth in biofilm. Generated colonies in cefmetazole plates were replicated onto fresh agar plates containing $4 \mu \mathrm{g} \mathrm{mL}-1$ cefmetazole to confirm the acquired resistance, and those that could grow were counted as transformants. Transformation frequency was calculated as the ratio of the number of transformants to the total c.f.u. Non-detected values were assigned half the value of the detection limit of the strain for the calculation of mean values (Figs. 2, 4) and statistical analyses.

\section{Stability test for cefoxitin resistance}

Glycerol stocks of mecA transformants were streaked on BHI plates supplemented with $4 \mu \mathrm{g} \mathrm{mL} \mathrm{m}^{-1}$ cefoxitin. Emerged single colonies were inoculated into BHI medium supplemented with $4 \mu \mathrm{g} \mathrm{mL}-1$ cefoxitin and grown with shaking at $37{ }^{\circ} \mathrm{C}$ for $12 \mathrm{~h}$. This culture was then diluted 1:1000 in drug-free BHI medium and grown for another $12 \mathrm{~h}$. After $12 \mathrm{~h}$ cells were plated on drug-free BHI agar and were replicated on BHI agar supplemented with $4 \mu \mathrm{g} \mathrm{mL} \mathrm{m}^{-1}$ cefoxitin to assess the population percentage that maintained growth ability under selective pressure from the $\beta$-lactam drug.

\section{Biofilm staining and quantification}

Biofilm formation was assessed in 96 -well plates ${ }^{65}$. Overnight cultures were diluted 1:200 in CS2 medium and $200 \mu \mathrm{l}$ were transferred to each well. Following $24 \mathrm{~h}$ of static 
incubation at $37{ }^{\circ} \mathrm{C}$, the nonadherent cells in medium were aspirated and the wells were stained with $200 \mu \mathrm{l}$ of $0.1 \%$ crystal violet for $15 \mathrm{~min}$. The wells were then gently washed 3 times with $200 \mu \mathrm{l}$ of PBS to remove residual stain before air drying. For biomass quantification, $100 \mu \mathrm{l}$ of $96 \%$ ethanol was added to the wells and incubated at room temperature for $10 \mathrm{~min}$ to solubilize the stain. The absorbance at $595 \mathrm{~nm}$ of the resolved stain was measured by plate reader.

To assess biofilm development in the natural transformation assay, the same cell suspension was inoculated in a 6-well plate containing CS2 medium ( $1.5 \mathrm{~mL}$ total volume per well). These plates were statically incubated for $96 \mathrm{~h}$ at $37^{\circ} \mathrm{C}$. Every $24 \mathrm{~h}$, formed biofilms were stained and quantified. For biofilm staining, the nonadherent cells were removed and

511 the wells were stained with $1.5 \mathrm{~mL}$ of $0.1 \%$ crystal violet for $15 \mathrm{~min}$. Excess stain was removed by distilled water and the wells were air dried. For biomass quantification, $750 \mu$ of $96 \%$ ethanol was added to the wells for $10 \mathrm{~min}$ at room temperature and the absorbance at $595 \mathrm{~nm}$ of the resolved stain was measured. Cell-free wells were used as blanks.

\section{SCCmec typing and long amplification by PCR} described $^{66}$. Long amplifications of SCCmec were performed using KOD One PCR master mix (TOYOBO) according to the manufacturer's instructions.

\section{Statistical analysis}

Statistical analyses were performed by GraphPad Prism (GraphPad Software, version 
The $\log$ values of natural transformation frequencies were analysed statistically. $\mathrm{P}$ values

below 0.05 were considered statistically significant.

\section{Acknowledgement}

532 This study was supported by Takeda Science Foundation, Pfizer Academic Contributions, JSPS KAKENHI Grant Number 25860313 and 18H02652 (to KM), Program to Disseminate Tenure Tracking System, MEXT (to RLO). We would like to express our appreciation to Dr. Teruyo Ito for valuable discussion, and Ms. Yoshimi Tsutsumi, Mr. Tin Ming Tan, Mr. Bobby Sookhoo, and Ms. Clara Effenberger for their experimental help. We would also like

\section{References}

1 Haaber, J., Penades, J. R. \& Ingmer, H. Transfer of Antibiotic Resistance in Staphylococcus aureus. Trends Microbiol 25, 893-905, doi:10.1016/j.tim.2017.05.011 (2017).

2 DeLeo, F. R., Otto, M., Kreiswirth, B. N. \& Chambers, H. F. Community-associated meticillin-resistant Staphylococcus aureus. Lancet 375, 1557-1568, doi:10.1016/S01406736(09)61999-1 (2010).

3 Ito, T., Okuma, K., Ma, X. X., Yuzawa, H. \& Hiramatsu, K. Insights on antibiotic resistance of Staphylococcus aureus from its whole genome: genomic island SCC. Drug Resist Updat 6 , 41-52 (2003).

4 Lowy, F. D. Antimicrobial resistance: the example of Staphylococcus aureus. J Clin Invest 111, 1265-1273, doi:10.1172/JCI18535 (2003).

5 Chambers, H. F. \& Deleo, F. R. Waves of resistance: Staphylococcus aureus in the antibiotic era. Nature reviews. Microbiology 7, 629-641, doi:10.1038/nrmicro2200 (2009).

6 Lee, A. S. et al. Methicillin-resistant Staphylococcus aureus. Nat Rev Dis Primers 4, 18033, doi:10.1038/nrdp.2018.33 (2018).

7 CDDEP. ResistanceMap:Antibiotic resistance., $<$ https://resistancemap.cddep.org/AntibioticResistance.php.> (2020).

8 Lindsay, J. A. Genomic variation and evolution of Staphylococcus aureus. Int J Med Microbiol 300, 98-103 (2010).

9 Malachowa, N. \& DeLeo, F. R. Mobile genetic elements of Staphylococcus aureus. Cell Mol Life Sci 67, 3057-3071, doi:10.1007/s00018-010-0389-4 (2010).

10 Katayama, Y., Ito, T. \& Hiramatsu, K. A new class of genetic element, staphylococcus cassette chromosome mec, encodes methicillin resistance in Staphylococcus aureus. Antimicrobial Agents and Chemotherapy 44, 1549 - 1555 (2000).

11 Robinson, D. A. \& Enright, M. C. Evolutionary models of the emergence of methicillinresistant Staphylococcus aureus. Antimicrob Agents Chemother 47, 3926-3934, 
doi:10.1128/aac.47.12.3926-3934.2003 (2003).

12 Morikawa, K. et al. A new staphylococcal sigma factor in the conserved gene cassette: functional significance and implication for the evolutionary processes. Genes Cells 8, 699-712 (2003).

13 Morikawa, K. et al. Expression of a cryptic secondary sigma factor gene unveils natural competence for DNA transformation in Staphylococcus aureus. PLoS Pathog 8, e1003003, doi:10.1371/journal.ppat.1003003 (2012).

14 Fagerlund, A., Granum, P. E. \& Havarstein, L. S. Staphylococcus aureus competence genes: mapping of the SigH, ComK1 and ComK2 regulons by transcriptome sequencing. Mol Microbiol 94, 557-579, doi:10.1111/mmi.12767 (2014).

15 McCarthy, A. J., Lindsay, J. A. \& Loeffler, A. Are all meticillin-resistant Staphylococcus aureus (MRSA) equal in all hosts? Epidemiological and genetic comparison between animal and human MRSA. Veterinary dermatology 23, 267-275, e253-264, doi:10.1111/j.13653164.2012.01072.x (2012).

16 Haag, A. F. \& Bagnoli, F. The Role of Two-Component Signal Transduction Systems in Staphylococcus aureus Virulence Regulation. Curr Top Microbiol Immunol 409, 145-198, doi:10.1007/82_2015_5019 (2017).

17 Villanueva, M. et al. Sensory deprivation in Staphylococcus aureus. Nat Commun 9, 523, doi:10.1038/s41467-018-02949-y (2018).

18 Dubrac, S. \& Msadek, T. Identification of genes controlled by the essential YycG/YycF twocomponent system of Staphylococcus aureus. J Bacteriol 186, 1175-1181 (2004).

19 Kuroda, M. et al. Two-component system VraSR positively modulates the regulation of cellwall biosynthesis pathway in Staphylococcus aureus. Mol Microbiol 49, 807-821 (2003).

20 Hiron, A., Falord, M., Valle, J., Debarbouille, M. \& Msadek, T. Bacitracin and nisin resistance in Staphylococcus aureus: a novel pathway involving the BraS/BraR twocomponent system (SA2417/SA2418) and both the BraD/BraE and VraD/VraE ABC transporters. Mol Microbiol 81, 602-622, doi:10.1111/j.1365-2958.2011.07735.x (2011).

21 Pietiainen, M. et al. Transcriptome analysis of the responses of Staphylococcus aureus to antimicrobial peptides and characterization of the roles of $v r a D E$ and vraSR in antimicrobial resistance. BMC Genomics 10, 429, doi:10.1186/1471-2164-10-429 (2009).

22 Gardete, S., Wu, S. W., Gill, S. \& Tomasz, A. Role of VraSR in antibiotic resistance and antibiotic-induced stress response in Staphylococcus aureus. Antimicrob Agents Chemother 50, 3424-3434, doi:10.1128/AAC.00356-06 (2006).

23 Kolar, S. L. et al. NsaRS is a cell-envelope-stress-sensing two-component system of Staphylococcus aureus. Microbiology 157, 2206-2219, doi:10.1099/mic.0.049692-0 (2011).

24 Geisinger, E., George, E. A., Chen, J., Muir, T. W. \& Novick, R. P. Identification of ligand specificity determinants in AgrC, the Staphylococcus aureus quorum-sensing receptor. J Biol Chem 283, 8930-8938, doi:10.1074/jbc.M710227200 (2008).

25 Redfield, R. J. Is quorum sensing a side effect of diffusion sensing? Trends Microbiol 10, 365370, doi:10.1016/s0966-842x(02)02400-9 (2002).

26 Shompole, S. et al. Biphasic intracellular expression of Staphylococcus aureus virulence factors and evidence for Agr-mediated diffusion sensing. Mol Microbiol 49, 919-927, doi:10.1046/j.1365-2958.2003.03618.x (2003).

27 Claverys, J. P., Martin, B. \& Polard, P. The genetic transformation machinery: composition, localization, and mechanism. FEMS Microbiol Rev 33, 643-656, doi:10.1111/j.15746976.2009.00164.x (2009).

28 Scharn, C. R., Tenover, F. C. \& Goering, R. V. Transduction of staphylococcal cassette chromosome mec elements between strains of Staphylococcus aureus. Antimicrob Agents Chemother 57, 5233-5238, doi:10.1128/AAC.01058-13 (2013).

29 Deghorain, M. \& Van Melderen, L. The Staphylococci phages family: an overview. Viruses 4, 3316-3335, doi:10.3390/v4123316 (2012).

30 Purohit, P. K. et al. Forces during bacteriophage DNA packaging and ejection. Biophys J 88, 851-866, doi:10.1529/biophysj.104.047134 (2005).

31 Ray, M., Boundy, S., Archer, GL. Transfer of the methicillin resistance genomic island among staphylococci by conjugation. Molecular Microbiology 100, 675 - 685 (2016).

32 Tsukubakishita, S., Kuwahara-Arai, K., Baba, T., Hiramatsu, K. Staphylococcal cassette chromosome mec-like element in Macrococcus caseolyticus. Antimicrobial Agents and Chemotherapy 54, 1469 - 1475 (2010). 
bioRxiv preprint doi: https://doi.org/10.1101/2020.12.08.415893; this version posted December 8,2020 . The copyright holder for this

preprint (which was not certified by peer review) is the author/funder. All rights reserved. No reuse allowed without permission.

33 Luong, T. T., Ouyang, S., Bush, K. \& Lee, C. Y. Type 1 capsule genes of Staphylococcus aureus are carried in a staphylococcal cassette chromosome genetic element. J Bacteriol 184, 3623-3629, doi:10.1128/jb.184.13.3623-3629.2002 (2002).

34 Kuroda, M. et al. Whole genome sequence of Staphylococcus saprophyticus reveals the pathogenesis of uncomplicated urinary tract infection. Proc Natl Acad Sci U S A 102, 1327213277 (2005).

35 Holden, M. T. et al. Complete genomes of two clinical Staphylococcus aureus strains: evidence for the rapid evolution of virulence and drug resistance. Proc Natl Acad Sci USA 101, 9786-9791, doi:10.1073/pnas.0402521101 (2004).

36 Lakhundi, S. \& Zhang, K. Methicillin-Resistant Staphylococcus aureus: Molecular Characterization, Evolution, and Epidemiology. Clin Microbiol Rev 31, doi:10.1128/CMR.00020-18 (2018).

37 Ito, T., Katayama, Y. \& Hiramatsu, K. Cloning and nucleotide sequence determination of the entire mec DNA of pre-methicillin-resistant Staphylococcus aureus N315. Antimicrob Agents Chemother 43, 1449-1458 (1999).

38 Ito, T. et al. Structural comparison of three types of staphylococcal cassette chromosome mec integrated in the chromosome in methicillin-resistant Staphylococcus aureus. Antimicrob Agents Chemother 45, 1323-1336, doi:10.1128/AAC.45.5.1323-1336.2001 (2001).

39 Ito, T., Ma, XX., Takekuchi, F., Okuma, K., Yuzawa, H., Hiramatsu, K. Novel type V staphylococcal cassette chromosome mec driven by a novel cassette chromosome recombinase,ccrC. Antimicrobial Agents and Chemotherapy 48, 2637 - 2651 (2004).

40 Ma, X., Ito, T., Tiensasitorn, C., Jamklnag, M., Chongtrakool, P., Boyle-Vavra, S., Daum, RS., Hiramatsu, K. Novel type of staphylococcal cassette chromosome mec identified in community-acquired methicillin-resistant Staphylococcus aureus strains. 464 (2002).

$41 \mathrm{Wu}$, S., Piscitelli, C., de Lencastre, H., Tomasz, A. Tracking the evolutionary origin of the methicillin resistance gene: cloning and sequencing of a homologue of mecA from a methicillin susceptible strain of Staphylococcus sciuri. Microb Drug Resist 2, 435 - 441 (1996).

42 Tsukubakishita, S., Kuwahara-Arai, K., Sasaki, T., Hiramatsu, K. Origin and molecular evolution of the determinant of methicillin resistance in staphylococci. Antimicrobial Agents and Chemotherapy 54, 4352 - 4359 (2010).

43 Stojanov, M., Sakwinska, O. \& Moreillon, P. Expression of SCC mec cassette chromosome recombinases in methicillin-resistant Staphylococcus aureus and Staphylococcus epidermidis. J Antimicrob Chemother 68, 749-757, doi:10.1093/jac/dks494 (2013).

44 Wang, L. \& Archer, G. L. Roles of CcrA and CcrB in excision and integration of staphylococcal cassette chromosome mec, a Staphylococcus aureus genomic island. J Bacteriol 192, 32043212, doi:10.1128/JB.01520-09 (2010).

45 Cohen, S. \& Sweeney, H. M. Transduction of Methicillin Resistance in Staphylococcus aureus Dependent on an Unusual Specificity of the Recipient Strain. J Bacteriol 104, 1158-1167, doi:10.1128/JB.104.3.1158-1167.1970 (1970).

46 Sjostrom, J. E., Lofdahl, S. \& Philipson, L. Transformation Reveals a Chromosomal Locus of Gene(S) for Methicillin Resistance in Staphylococcus-Aureus. Journal of Bacteriology 123, 905-915 (1975).

47 Maslanova, I. et al. Bacteriophages of Staphylococcus aureus efficiently package various bacterial genes and mobile genetic elements including SCCmec with different frequencies. Environ Microbiol Rep 5, 66-73, doi:10.1111/j.1758-2229.2012.00378.x (2013).

48 Chlebowicz, M. A. et al. The Staphylococcal Cassette Chromosome mec type V from Staphylococcus aureus ST398 is packaged into bacteriophage capsids. Int J Med Microbiol 304, 764-774, doi:10.1016/j.ijmm.2014.05.010 (2014).

49 Ender, M., McCallum, N., Adhikari, R. \& Berger-Bachi, B. Fitness cost of SCCmec and methicillin resistance levels in Staphylococcus aureus. Antimicrob Agents Chemother 48, 2295-2297, doi:10.1128/AAC.48.6.2295-2297.2004 (2004).

50 de Lencastre, H. \& Tomasz, A. Reassessment of the number of auxiliary genes essential for expression of high-level methicillin resistance in Staphylococcus aureus. Antimicrob Agents Chemother 38, 2590-2598, doi:10.1128/aac.38.11.2590 (1994).

51 Miragaia, M. Factors Contributing to the Evolution of mecA-Mediated beta-lactam Resistance in Staphylococci: Update and New Insights From Whole Genome Sequencing (WGS). Frontiers in Microbiology 9, doi:ARTN 272310.3389/fmicb.2018.02723 (2018).

52 Katayama, Y., Zhang, H. Z., Hong, D. \& Chambers, H. F. Jumping the barrier to beta-lactam 
resistance in Staphylococcus aureus. J Bacteriol 185, 5465-5472, doi:10.1128/jb.185.18.54655472.2003 (2003).

53 Moormeier, D. E., Bose, J. L., Horswill, A. R. \& Bayles, K. W. Temporal and Stochastic Control of Staphylococcus aureus Biofilm Development. mBio 5, doi:ARTN e013411410.1128/mBio.01341-14 (2014).

54 Kavanaugh, J. S. et al. Identification of Extracellular DNA-Binding Proteins in the Biofilm Matrix. mBio 10, doi:ARTN e01137-1910.1128/mBio.01137-19 (2019).

55 Moormeier, D. E. \& Bayles, K. W. Staphylococcus aureus biofilm: a complex developmental organism. Molecular Microbiology 104, 365-376, doi:10.1111/mmi.13634 (2017).

56 Moreno-Gamez, S. et al. Quorum sensing integrates environmental cues, cell density and cell history to control bacterial competence. Nature Communications 8, doi:ARTN 85410.1038/s41467-017-00903-y (2017).

57 Paharik, A. E. \& Horswill, A. R. The Staphylococcal Biofilm: Adhesins, Regulation, and Host Response. Microbiology spectrum 4, doi:10.1128/microbiolspec.VMBF-0022-2015 (2016).

58 Costerton, J. W., Stewart, P. S. \& Greenberg, E. P. Bacterial biofilms: A common cause of persistent infections. Science 284, 1318-1322, doi:DOI 10.1126/science.284.5418.1318 (1999).

59 Otto, M. MRSA virulence and spread. Cellular microbiology 14, 1513-1521, doi:10.1111/j.1462-5822.2012.01832.x (2012).

60 Savage, V. J., Chopra, I. \& O'Neill, A. J. Staphylococcus aureus Biofilms Promote Horizontal Transfer of Antibiotic Resistance. Antimicrobial Agents and Chemotherapy 57, 1968-1970, doi:10.1128/Aac.02008-12 (2013).

61 Resch, A., Fehrenbacher, B., Eisele, K., Schaller, M. \& Gotz, F. Phage release from biofilm and planktonic Staphylococcus aureus cells. Fems Microbiology Letters 252, 89-96, doi:10.1016/j.femsle.2005.08.048 (2005).

62 Tao, L., Wu, X. \& Sun, B. Alternative sigma factor sigmaH modulates prophage integration and excision in Staphylococcus aureus. PLoS Pathog 6, e1000888 (2010).

63 Arnaud, M., Chastanet, A. \& Debarbouille, M. New vector for efficient allelic replacement in naturally nontransformable, low-GC-content, gram-positive bacteria. Appl Environ Microbiol 70, 6887-6891 (2004).

64 Tsai, M. et al. Staphylococcus aureus requires cardiolipin for survival under conditions of high salinity. BMC Microbiol 11, 13, doi:10.1186/1471-2180-11-13 (2011).

65 Christensen, G. D. et al. Adherence of Coagulase-Negative Staphylococci to Plastic TissueCulture Plates - a Quantitative Model for the Adherence of Staphylococci to Medical Devices. Journal of Clinical Microbiology 22, 996-1006, doi:Doi 10.1128/Jcm.22.6.996-1006.1985 (1985).

66 Ghaznavi-Rad, E., Shamsudin, M. N., Sekawi, Z., van Belkum, A. \& Neela, V. A simplified multiplex PCR assay for fast and easy discrimination of globally distributed staphylococcal cassette chromosome mec types in meticillin-resistant Staphylococcus aureus. Journal of medical microbiology 59, 1135-1139, doi:10.1099/jmm.0.021956-0 (2010).

67 Martin, P. K., Li, T., Sun, D., Biek, D. P. \& Schmid, M. B. Role in cell permeability of an essential two-component system in Staphylococcus aureus. J Bacteriol 181, 3666-3673 (1999).

68 Park, J. Y. et al. Characterization of a Novel Two-Component Regulatory System, HptRS, the Regulator for the Hexose Phosphate Transport System in Staphylococcus aureus. Infection and Immunity 83, 1620-1628, doi:10.1128/Iai.03109-14 (2015).

69 Brunskill, E. W. \& Bayles, K. W. Identification of LytSR-regulated genes from Staphylococcus aureus. J Bacteriol 178, 5810-5812 (1996).

70 Yang, S. J. et al. Role of the LytSR two-component regulatory system in adaptation to cationic antimicrobial peptides in Staphylococcus aureus. Antimicrob Agents Chemother 57, 3875-3882, doi:10.1128/AAC.00412-13 (2013).

71 Patton, T. G., Yang, S. J. \& Bayles, K. W. The role of proton motive force in expression of the Staphylococcus aureus cid and Irg operons. Mol Microbiol 59, 1395-1404, doi:10.1111/j.13652958.2006.05034.x (2006).

72 Cui, L., Lian, J. Q., Neoh, H. M., Reyes, E. \& Hiramatsu, K. DNA microarray-based identification of genes associated with glycopeptide resistance in Staphylococcus aureus. Antimicrob Agents Chemother 49, 3404-3413 (2005).

73 Falord, M., Mader, U., Hiron, A., Debarbouille, M. \& Msadek, T. Investigation of the Staphylococcus aureus GraSR regulon reveals novel links to virulence, stress response and cell wall signal transduction pathways. PLoS One 6, e21323, doi:10.1371/journal.pone.0021323 (2011). 
74 Giraudo, A. T., Calzolari, A., Cataldi, A. A., Bogni, C. \& Nagel, R. The sae locus of Staphylococcus aureus encodes a two-component regulatory system. FEMS Microbiol Lett 177, 15-22 (1999).

75 Novick, R. P. \& Jiang, D. The staphylococcal saeRS system coordinates environmental signals with agr quorum sensing. Microbiology 149, 2709-2717, doi:10.1099/mic.0.26575-0 (2003).

76 Fournier, B. \& Hooper, D. C. A new two-component regulatory system involved in adhesion, autolysis, and extracellular proteolytic activity of Staphylococcus aureus. J Bacteriol 182, 3955-3964 (2000).

77 Liang, X. et al. Global regulation of gene expression by ArlRS, a two-component signal transduction regulatory system of Staphylococcus aureus. J Bacteriol 187, 5486-5492, doi:10.1128/JB.187.15.5486-5492.2005 (2005).

78 Walker, J. N. et al. The Staphylococcus aureus ArlRS two-component system is a novel regulator of agglutination and pathogenesis. PLoS Pathog 9, e1003819, doi:10.1371/journal.ppat.1003819 (2013).

79 Yarwood, J. M., McCormick, J. K. \& Schlievert, P. M. Identification of a novel two-component regulatory system that acts in global regulation of virulence factors of Staphylococcus aureus. J Bacteriol 183, 1113-1123, doi:10.1128/JB.183.4.1113-1123.2001 (2001).

80 Birkey, S. M., Liu, W., Zhang, X., Duggan, M. F. \& Hulett, F. M. Pho signal transduction network reveals direct transcriptional regulation of one two-component system by another two-component regulator: Bacillus subtilis $\mathrm{PhoP}$ directly regulates production of ResD. Mol Microbiol 30, 943-953 (1998).

81 Kelliher, J. L., Radin, J. N. \& Kehl-Fie, T. E. PhoPR Contributes to Staphylococcus aureus Growth during Phosphate Starvation and Pathogenesis in an Environment-Specific Manner. Infection and Immunity 86, doi:ARTN e00371-1810.1128/IAI.00371-18 (2018).

82 Sun, F. et al. AirSR, a [2Fe-2S] cluster-containing two-component system, mediates global oxygen sensing and redox signaling in Staphylococcus aureus. Journal of the American Chemical Society 134, 305-314, doi:10.1021/ja2071835 (2012).

83 Lina, G. et al. Transmembrane topology and histidine protein kinase activity of AgrC, the agr signal receptor in Staphylococcus aureus. Mol Microbiol 28, 655-662 (1998).

84 Novick, R. P. \& Geisinger, E. Quorum sensing in staphylococci. Annu Rev Genet 42, 541-564, doi:10.1146/annurev.genet.42.110807.091640 (2008).

85 Freeman, Z. N., Dorus, S. \& Waterfield, N. R. The KdpD/KdpE two-component system: integrating $\mathrm{K}(+)$ homeostasis and virulence. PLoS Pathog 9, e1003201, doi:10.1371/journal.ppat.1003201 (2013).

86 Torres, V. J. et al. A Staphylococcus aureus regulatory system that responds to host heme and modulates virulence. Cell host \& microbe 1, 109-119, doi:10.1016/j.chom.2007.03.001 (2007).

87 Schlag, S. et al. Characterization of the oxygen-responsive NreABC regulon of Staphylococcus aureus. J Bacteriol 190, 7847-7858, doi:10.1128/JB.00905-08 (2008).

88 Kuwahara-Arai, K., Kondo, N., Hori, S., Tateda-Suzuki, E. \& Hiramatsu, K. Suppression of methicillin resistance in a mecA-containing pre-methicillin-resistant Staphylococcus aureus strain is caused by the mecI-mediated repression of PBP 2' production. Antimicrob Agents Chemother 40, 2680-2685 (1996).

89 Inose, Y. et al. Genetic characterization of the natural SigB variants found in clinical isolates of Staphylococcus aureus. J Gen Appl Microbiol 52, 259-271 (2006).

90 Kreiswirth, B. N. et al. The toxic shock syndrome exotoxin structural gene is not detectably transmitted by a prophage. Nature 305, 709-712 (1983). 


\section{Captions}

Figure 1. TCS12, TCS13 and TCS17 are involved in com $G$ promoter activity. a, 17 TCSs in $S$. aureus N315. Gene name and locus in N315 genome are shown. b,c, $\Delta$ TCS mutants $(\Delta 3$ - $\Delta 17)$ were tested for involvement in the regulation of $\mathrm{P}_{c o m G}$. Nef and its derivative $\Delta T C S$ carrying the $\mathrm{P}_{c o m G}-g f p$ reporter were grown in CS2 medium with shaking. b, The $\mathrm{Y}$ axis shows the increase in RFU/OD 600 values, which was calculated by subtracting the minimum GFP intensities from the maximum GFP intensities during 12-24 h of growth. The mean of $\mathrm{n}=3-8$ independent experiments are shown. Error bars represent s.d. Statistical significance was determined by one-way ANOVA with Dunnett's multiple comparison test. ${ }^{*} \mathrm{P}<0.05$, ${ }^{* *}$ $\mathrm{P}<0.01,{ }^{* * *} \mathrm{P}<0.001$. c, The population percentage expressing GFP was determined after 12-14 $\mathrm{h}$ of growth by fluorescent microscopy (bottom). The mean of $n=4-9$ independent experiments are shown. Error bars represent s.d. Statistical significance was determined by one-way ANOVA with Tukey's multiple comparison test. ${ }^{*} \mathrm{P}<0.05$, ${ }^{* *} \mathrm{P}<0.01,{ }^{* * *} \mathrm{P}<$ $0.001,{ }^{* * * *} \mathrm{P}<0.0001$. Scale bars, $5 \mu \mathrm{m}$. d, Schematic summary of the TCSs involved in $\mathrm{P}_{\text {comG }}$ regulation.

Figure 2. TCSs 13 and 17 are important for $\operatorname{com} G$ promoter activity and natural transformation in biofilm growth conditions. a, The percentage of $\mathrm{Nef}$ and its derivatives expressing $\mathrm{P}_{c o m G-} g f p$ reporter. Cells were statically grown in CS2 medium. The mean $\mathrm{n}=3-10$. Error bars represent s.d. b, Chromosomal complementation of the mutants restores the percentage of cells expressing GFP at day 3 in the biofilm. The mean $n=7-10$. Error bars represent s.d. c, Transformation frequencies of Nef (red) and Nefh (blue) at day 3 in the biofilm growth condition compared with the planktonic growth condition. Transformants were selected by tetracycline. Dotted line represent detection limit of planktonic Nef. d, Time-course development of natural transformation in the biofilm. Nef and its derivatives were statically grown in CS2 medium. Natural transformation frequencies were determined every $24 \mathrm{~h}$. Dotted lines represent the detection limit. Data points represent independent experiments. Error bars represent s.d. e, Complementation of the TCS13 and TCS17 restores the transformation frequencies at day 3 in the biofilm. In this experiment, chromosomally complemented strains were used because plasmid-based complimented strains are tetracycline resistant. Data points represent independent experiments. Error bars represent s.d. Statistical significance $(\mathbf{b}, \mathbf{e})$ was determined by one-way ANOVA with Tukey's multiple comparison test. ${ }^{*} \mathrm{P}<0.05,{ }^{* *} \mathrm{P}<0.01,{ }^{* * * *} \mathrm{P}<0.0001$.

Figure 3. SCCmec transformation. a, Presence of the mecA gene was verified by PCR using mecAF and mecAR primers. Chromosomal DNA of donor and recipients were used for positive and negative controls. $\mathbf{b}$, Stability of resistance. The transformants were passaged in drug-free media for $12 \mathrm{~h}$ after growth with cefoxitin $\left(4 \mu \mathrm{g} \mathrm{mL} \mathrm{mL}^{-1}\right)$ for $12 \mathrm{~h}$ (Time 0$)$. The percentage of cells that can grow on cefoxitin was calculated by a replica method. The mean of $n=2$ independent experiments is shown. Error bars represent s.d. c, Disk diffusion test of $\beta$-lactam antibiotics. CFX: cefoxitin, MPI: oxacillin. right bottom: diameters of inhibitory zones. d,e, Schematic structures of SCCmec IVa in MW2 chromosomal DNA (d) and of SCCme II in N315 chromosomal DNA (e). Primer locations are indicated with arrows. Chromosomal DNA of MR-donors and MS-recipients were used for positive and negative controls. Suffix (1), (2) represents transformants obtained from two independent experiments. (d-e, bottom) Long PCR verification of the entire SCCmec IVa (d) or II (e) elements in all transformants where mecA was present. M: $\lambda$ HindIII. f, Intactness and stability of SCCmec in transformants. 
Figure 4. Bacitracin blocks natural transformation. Cells were grown with bacitracin (a), vancomycin (b), or nisin (c) under biofilm conditions in CS2 medium. Transformants were counted after 3 days. Error bars represent s.d. Dotted line represents the detection limit. Statistical significance was determined by Student's t-test. $* \mathrm{P}<0.05$.

Supplementary materials

Supplementary Table 1. Bacterial strains and plasmids used in this study.

\section{Supplementary Table 2. List of primers used in this study}

Supplementary Table 3. MIC $\left(\mu \mathrm{g} \mathrm{mL}^{-1}\right)$ of cell wall-targeting antibiotics in CS2 medium or in TSB.

\section{Supplementary Table 4. Intra- and interspecies transformation of distinct SCCmec elements under biofilm growth conditions.}

Supplementary Table 5. MIC $\left(\mu \mathrm{g} \mathrm{mL}^{-1}\right)$ of cefmetazole and cefoxitin in TSB.

Supplementary Figure 1. Reporter assay for $\operatorname{com} G$ promoter activity. Nef carrying the $\mathrm{P}_{c o m G}-g f p$ reporter was grown in either CS2 medium (a) or TSB (b) with shaking. Fluorescence intensity (green line) and $\mathrm{OD}_{600}$ (blue line) were measured every $30 \mathrm{~min}$. The mean of $\mathrm{n}=3$ independent experiments is shown. Error bars represent s.d.

Supplementary Figure 2. Growth curves of Nef and its derivative $\Delta$ TCS. Cells were grown in either TSB (a) or CS2 medium (b) with shaking. OD 600 was measured every $30 \mathrm{~min}$. The mean of $n=3$ independent experiments is shown. Error bars are omitted for clarity.

\section{Supplementary Figure 3. Biofilm formation is impaired in the TCS13 and TCS17} mutants.

(a) Nef and its derivatives were statically grown in CS2 medium in a 96-well plate for $24 \mathrm{~h}$. (b) Time-course of biofilm development in Nef and its derivatives. The cells were statically grown in CS2 medium in a 6-well plate for up to 4 days. (b, middle) The biofilms were stained with crystal violet and the absorbance at $595 \mathrm{~nm}$ was measured. The mean of $\mathrm{n}=2-3$ independent experiments is shown. Error bars represent SD. (b, right) CFU of the cells after $24 \mathrm{~h}$ in the biofilm.

Supplementary Figure 4. $\operatorname{com} G$ promoter activity is affected by cell wall-targeting antibiotics. Nef-GFP was treated with subinhibitory concentrations of vancomycin (a), bacitracin (b), or nisin (c). Cells were grown in CS2 medium with shaking for $24 \mathrm{~h}$. Fluorescence (RFU) and $\mathrm{OD}_{600}$ were measured every $30 \mathrm{~min}$. Data shown are either relative RFU/OD 600 values (left panels) or increases in RFU/OD 600 values during 8-24 h of growth (right panels).

Supplementary Figure 5. comG promoter activity is affected by multiple TCSs in

biofilm. Nef and its derivative $\Delta$ TCSs carrying the $\mathrm{P}_{c o m G}-g f p$ reporter were statically grown in CS2 medium in biofilm growth conditions for 3 days. The percentage of GFP-expressing cells was calculated as observed by fluorescence microscopy. The mean of $n=3-7$ independent experiments is shown. Error bars represent s.d. Statistical significance was 
determined by one-way ANOVA with Tukey's multiple comparison test. ${ }^{*} \mathrm{P}<0.05$, ${ }^{* * * *} \mathrm{P}<0.0001$.

Supplementary Figure 6. Nisin does not induce natural transformation in Nef. Nef and its derivatives were grown in CS2 medium with or without nisin $\left(8 \mu \mathrm{g} \mathrm{mL} \mathrm{mL}^{-1}\right)$. Transformation frequencies were determined after $10 \mathrm{~h}$ of planktonic growth. The transformants were selected by tetracycline. The mean of two independent experiments is shown with s.d. ND < $10^{-9}$.

Supplementary Figure 7. Nutrient-poor culture media are preferable for natural transformation under biofilm growth conditions. Nef, Nef-h, and Nef- $\Delta$ comE were statically grown in different growth media, including CS2, TSB, BHI, RPMI, and M9 supplemented with amino acids (M9+aa). Transformation frequencies were determined at day 3. Dotted lines represent the detection limit of the strains in each growth medium. The mean and s.d. are shown. Circles represent independent experiments. S. aureus and its derivative carrying the SigH expression plasmid pRIT-sigH (WT-h) were tested for transformation after 2 days of static growth in CS2 medium. The transformants were selected by tetracycline. The mean of $n=2-3$ independent experiments is shown. Error bars represent s.d. 
bioRxiv preprint doi: https://doi.org/10.1101/2020.12.08.415893; this version posted December 8, 2020. The copyright holder for this

preprint (which was not certified by peer review) is the author/funder. All rights reserved. No reuse allowed without permission.

Figure 1.

a

\begin{tabular}{|c|c|c|c|c|}
\hline & Gene name & $\begin{array}{l}\text { Gene locus } \\
\text { (SA number in N315) }\end{array}$ & Function/ Signal & References \\
\hline TCS1 & walKR & SA0018-SA0017 & Membrane permeability, cell wall metabolism, autolysis & 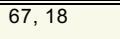 \\
\hline TCS2 & $k d p D E$ & SA0067-SA0066 & $\mathrm{K}^{+}$transport, virulence-related regulation, stress resistance & 85 \\
\hline TCS3 & hptRS & SA0215-SA0216 & Hexose-phosphate transport & 68 \\
\hline TCS4 & IytSR & SA0250-SA0251 & Autolysis, membrane electrical potential sensor, adaptation to cationic antimicrobial peptides & $69,71,70$ \\
\hline TCS5 & grasR & SA0615-SA0614 & CAMPs resistance, virulence, stress response, cell wall signaling, growth at acidic $\mathrm{pH}$ & $72,73,17$ \\
\hline TCS6 & saeSR & SA0660-SA0661 & Regulation of exoprotein expression, virulence & 74,75 \\
\hline TCS7 & & SA1158-SA1159 & Unknown & \\
\hline TCS8 & arlSR & SA1246-SA1248 & Autolysis, cell growth, agglutination, pathogenesis & $76,77,78$ \\
\hline TCs9 & srrBA & SA1322-SA1323 & Respiratory response, virulence & 79 \\
\hline TCS10 & phoRP & SA1515-SA1516 & Phosphate starvation response (in B. subtillis); Growth during phosphate starvation & 80,81 \\
\hline TCS11 & airSR & SA1667-SA1666 & Oxygen sensing and redox signaling & 82 \\
\hline TCS12 & $\operatorname{vraSR}$ & SA1701-SA1700 & Vancomycin resistance; response to cell wall-targeting antibiotics & 19 \\
\hline TCS13 & $\operatorname{agrCA}$ & SA1843-SA1844 & Quorum sensing, virulence & 83,84 \\
\hline TCS14 & $k d p D E$ & SA1882-SA1883 & $\mathrm{K}^{+}$transport, virulence-related regulation, stress resistance & 85 \\
\hline TCS15 & hssSR & SA2152-SA2151 & Heme sensor system, virulence & 86 \\
\hline TCS16 & nre $C B$ & SA2180-SA2179 & Nitrite and nitrate reduction and transport & 87 \\
\hline TCS17 & brasR & SA2417-SA2418 & Bacitracin and nisin resistance & 20 \\
\hline
\end{tabular}

b

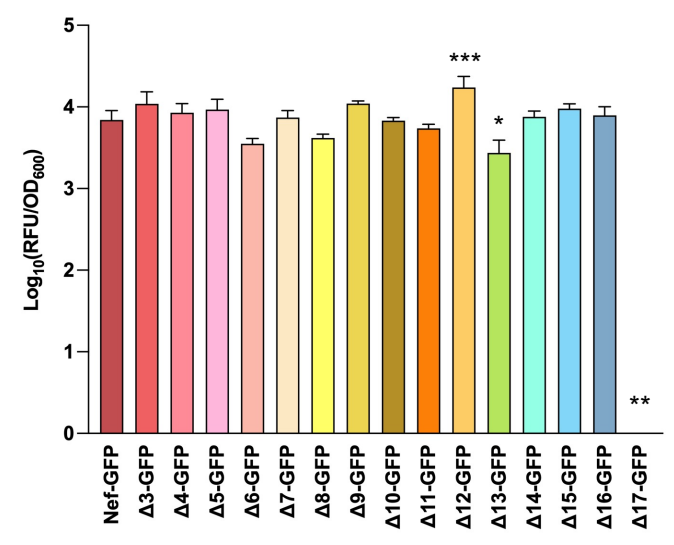

d

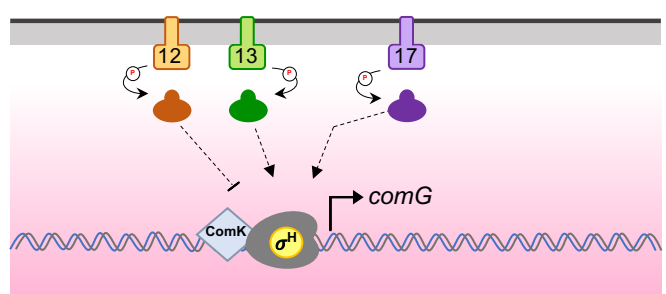

C
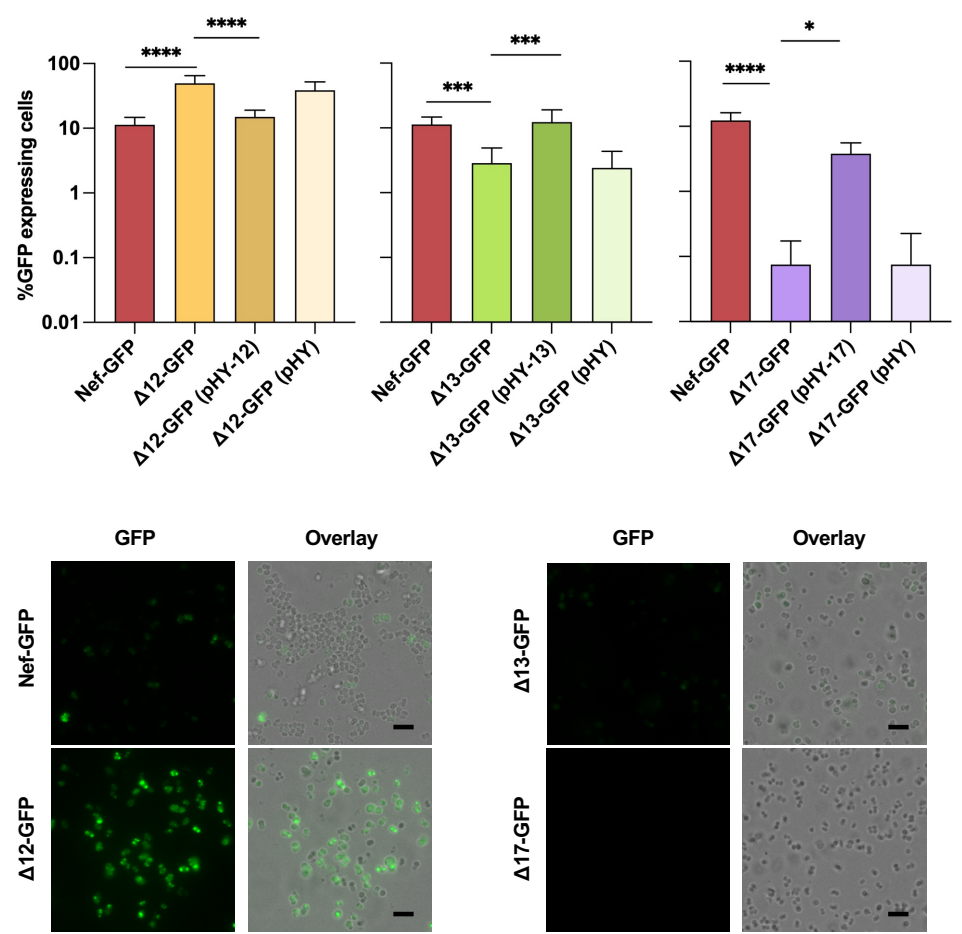

Overlay
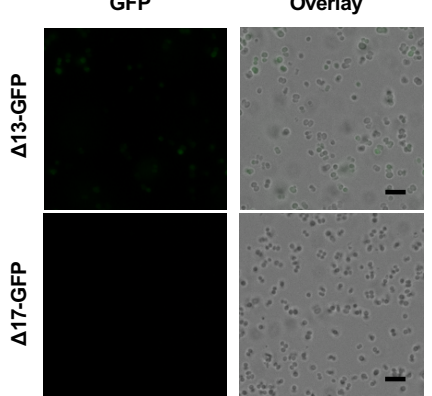

Figure 1. TCS12, TCS13 and TCS17 are involved in comG promoter activity. a, 17 TCSs in S. aureus N315. Gene name and locus in N315 genome are shown. b,c, $\Delta$ TCS mutants $(\Delta 3-\Delta 17)$ were tested for involvement in the regulation of $\mathrm{P}_{\text {comG. }}$ Nef and its derivative $\triangle$ TCS carrying the $\mathrm{P}_{\text {comG }}$-gfp reporter were grown in CS2 medium with shaking. $\mathbf{b}$, The $\mathrm{Y}$ axis shows the increase in RFU/OD 600 values, which was calculated by subtracting the minimum GFP intensities from the maximum GFP intensities during 12-24 $\mathrm{h}$ of growth. The mean of $\mathrm{n}=3-8$ independent experiments are shown. Error bars represent s.d. Statistical significance was determined by one-way ANOVA with Dunnett's multiple comparison test. * $P<0.05$, ${ }^{* *} \mathrm{P}<0.01,{ }^{* * *} \mathrm{P}<0.001$. c, The population percentage expressing GFP was determined after $12-14 \mathrm{~h}$ of growth by fluorescent microscopy (bottom). The mean $n=4-9$ independent experiments are shown. Error bars represent s.d. Statistical significance was determined by one-way ANOVA with Tukey's multiple comparison test. ${ }^{*} \mathrm{P}<0.05,{ }^{* *} \mathrm{P}<0.01,{ }^{* * *} \mathrm{P}<0.001$, ${ }^{* * *} \mathrm{P}<0.0001$. Scale bars, $5 \mu \mathrm{m}$. d, Schematic summary of the TCSs involved in $\mathrm{P}_{\text {com }}$ regulation. 
Figure 2.

a

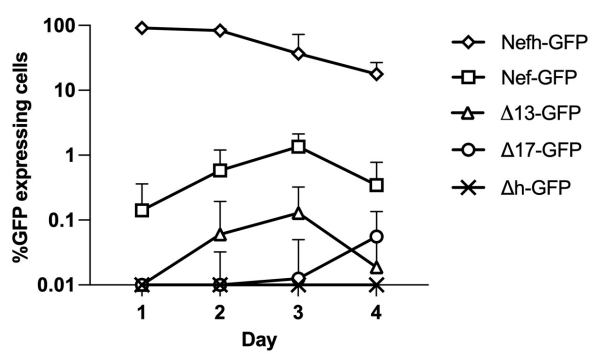

C

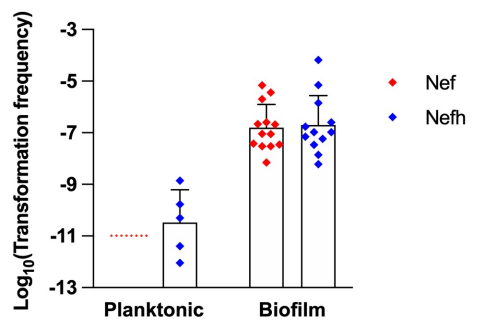

b

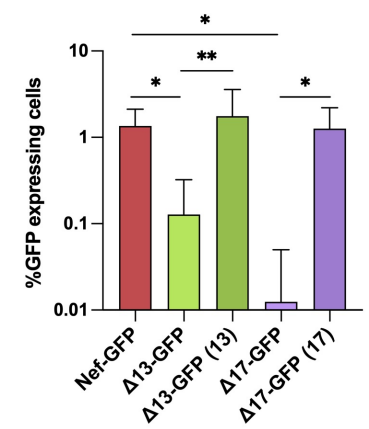

e

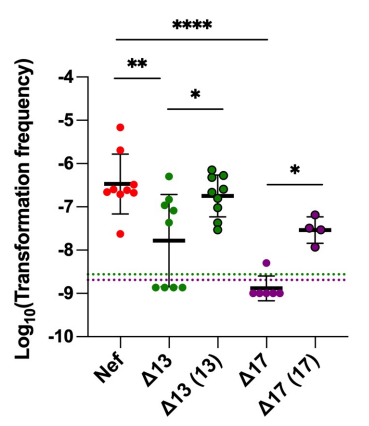

$\Delta 13$

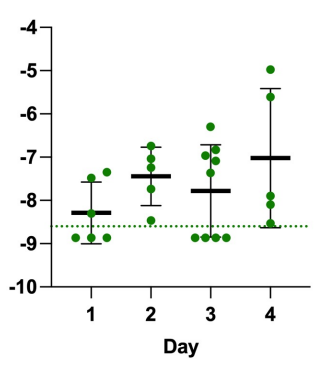

Nef $\Delta$ comE

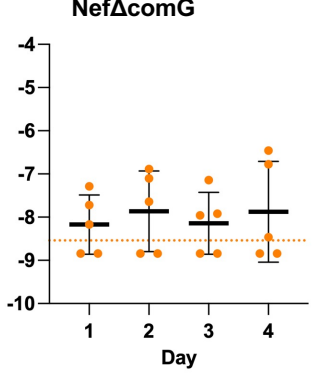

Figure 2. TCSs 13 and 17 are important for comG promoter activity and natural transformation in biofilm growth conditions. a, The percentage of Nef and its derivatives expressing $\mathrm{P}_{\text {comG-gfp reporter. Cells were statically }}$ grown in CS2 medium. The mean $n=3-$ 10. Error bars represent s.d. b, Chromosomal complementation of the mutants restores the percentage of cells expressing GFP at day 3 in the biofilm. The mean $n=7-10$. Error bars represent s.d. c, Transformation frequencies of Nef (red) and Nefh (blue) at day 3 in the biofilm growth condition compared with the planktonic growth condition. Transformants were selected by tetracycline. Dotted line represent detection limit of planktonic Nef. d, Timecourse development of natural transformation in the biofilm. Nef and its derivatives were statically grown in CS2 medium. Natural transformation frequencies were determined every $24 \mathrm{~h}$. Dotted lines represent the detection limit. Data points represent independent experiments. Error bars represent s.d. e, Complementation of the TCS13 and TCS17 restores the transformation frequencies at day 3 in the biofilm. In this experiment, chromosomally complemented strains were used because plasmid-based complimented strains are tetracycline resistant. Data points represent independent experiments. Error bars represent s.d. Statistical significance (b, e) was determined by one-way ANOVA with Tukey's multiple comparison test. ${ }^{*} \mathrm{P}<0.05,{ }^{* *} \mathrm{P}<0.01,{ }^{* * * *} \mathrm{P}<0.0001$. 
Figure 3.

a

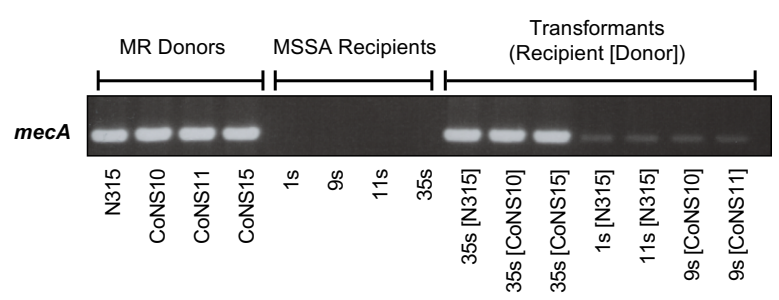

C

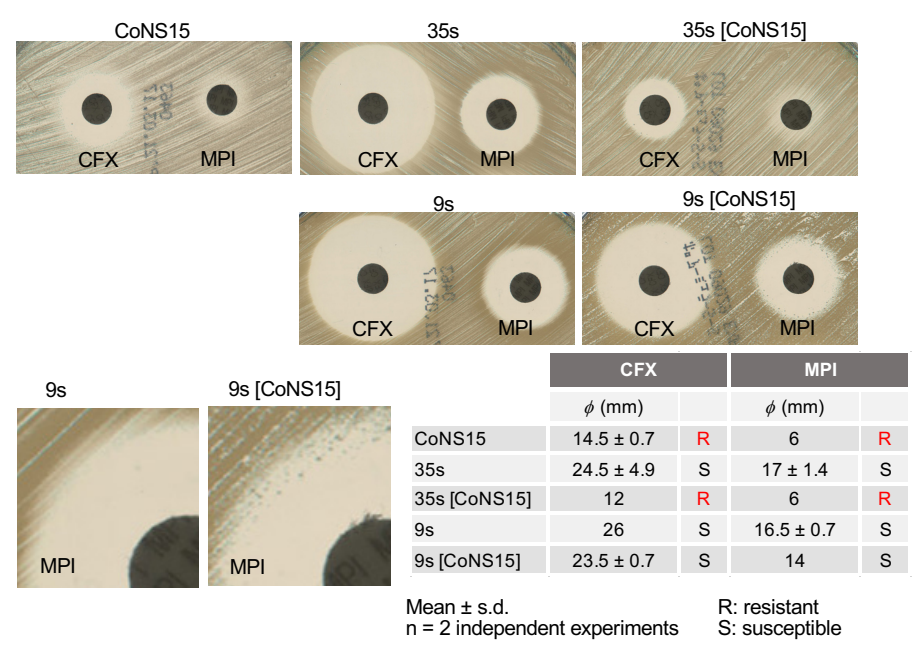

b

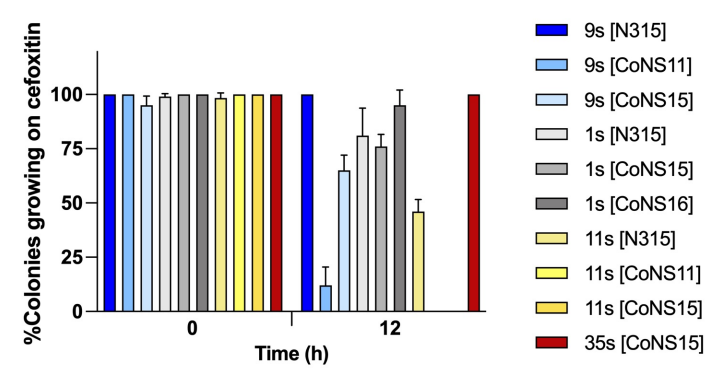

f

\begin{tabular}{|c|c|c|c|c|c|c|}
\hline \multirow[b]{2}{*}{ Donor } & \multirow[b]{2}{*}{$\begin{array}{l}\text { SCC } \\
\text { type }\end{array}$} & \multicolumn{5}{|c|}{ Recipient } \\
\hline & & Nef & $1 \mathrm{~s}$ & $9 \mathrm{~s}$ & $11 \mathrm{~s}$ & $35 \mathrm{~s}$ \\
\hline \multirow{2}{*}{ N315 } & \multirow{2}{*}{11} & Stable & Unstable & Stable & Unstable & Stable \\
\hline & & Full Length & & Full Length & & Rearragement \\
\hline \multirow{2}{*}{ CoNS11 } & \multirow{2}{*}{ IVa } & & & Unstable & Unstable & Stable \\
\hline & & & & & & Full Length \\
\hline \multirow{2}{*}{ CoNS15 } & \multirow{2}{*}{ IVa } & & Unstable & Unstable & Unstable & Stable \\
\hline & & & & & & Full Length \\
\hline \multirow{2}{*}{$\begin{array}{l}\text { CoNS16 } \\
\text { CoNS9 } \\
\text { CoNS10 } \\
\text { CoNS17 } \\
\text { MW2 }\end{array}$} & \multirow{2}{*}{\begin{tabular}{|l} 
III \\
III \\
IVa \\
IVa \\
IVa
\end{tabular}} & & & & & Stable \\
\hline & & & & & & Full Length \\
\hline
\end{tabular}

Stable: $m e c A$ is stably maintained

Unstable: mecA dissapears in the absence of antibiotics pressure Full length: Transferred SCC is full length Rearrangement: Mobile elements excised

d
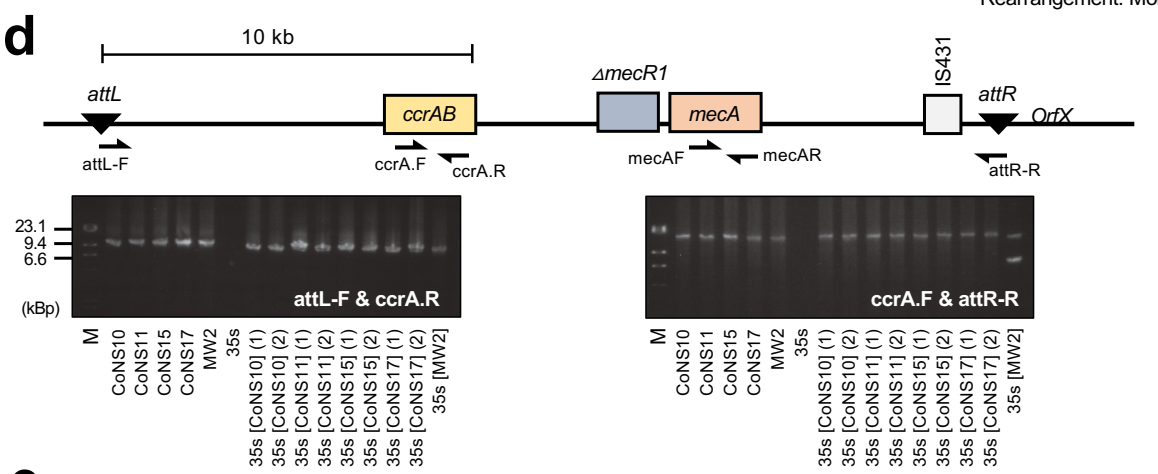

e
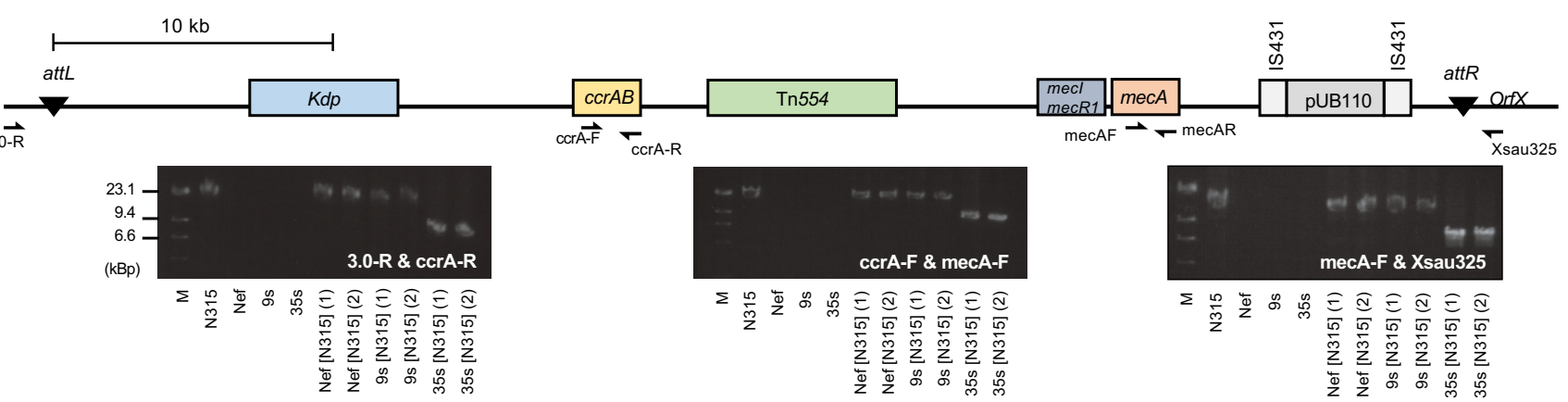

Figure 3. SCCmec transformation. a, Presence of the mecA gene was verified by PCR using mecAF and mecAR primers. Chromosomal DNA of donor and recipients were used for positive and negative controls. b, Stability of resistnce. The transformants were passaged in drug-free media for $12 \mathrm{~h}$ after growth with cefoxitin $\left(4 \mu \mathrm{g} \mathrm{mL} \mathrm{m}^{-1}\right)$ for $12 \mathrm{~h}($ Time 0$)$. The percentage of cells that can grow on cefoxitin was calculated by a replica method. The mean of $n=2$ independent experiments is shown. Error bars represent s.d. c, Disk diffusion test of $\beta$-lactam antibiotics. CFX: cefoxitin, MPI: oxacillin. d,e, Schematic structures of SCCmec IVa in MW2 chromosomal DNA (d) and of SCCme II in N315 chromosomal DNA (e). Primer locations are indicated with arrows. Chromosomal DNA of MR-donors and MS-recipients were used for positive and negative controls. Suffix (1), (2) represents transformants obtained from two independent experiments. (d-e, bottom) Long PCR verification of the entire SCCmec IVa (d) or II (e) elements in all transformants where mecA was present. M: $\lambda$ HindlII. f, Intactness and stability of SCCmec in transformants. 
Figure 4.

a

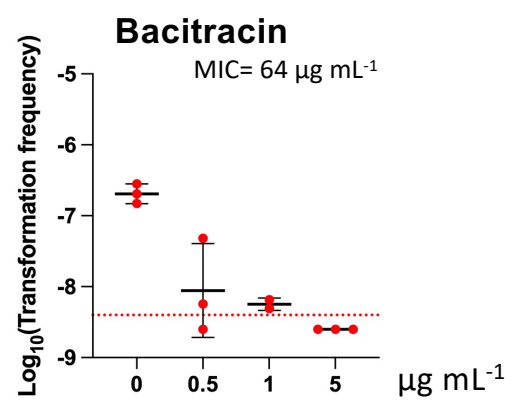

C

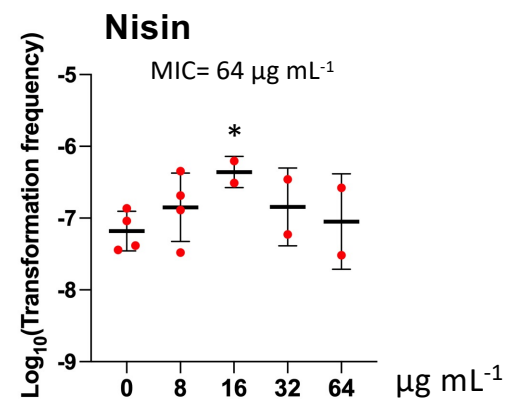

b

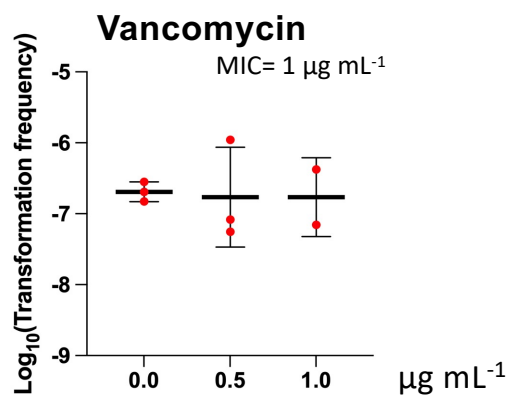

Figure 4. Bacitracin blocks natural transformation. Cells were grown with bacitracin (a), vancomycin (b), or nisin (c) under biofilm conditions in CS2 medium. Transformants were counted after 3 days. Error bars represent s.d. Statistical significance was determined by Student's t-test. ${ }^{*} \mathrm{P}<0.05$. 